\title{
Características de la ventilación mecánica invasiva en COVID-19 para médicos no especialistas
}

\author{
Characteristics of invasive mechanical ventilation in \\ COVID-19 for non-specialist medical
}

Bastian Abarca Rozas ${ }^{b}$, Jocelyn Vargas Urra E.Ua, Javier García Garzón

\begin{abstract}
SARS-CoV-2 is the agent responsible for COVID-19, the current pandemic, which is characterized by developing respiratory disturbances that are associated with severe hypoxemia associated with symptoms of non-bacterial pneumonia, ARDS up to multi-organ failure. It has been characterized by presenting 2 different phenotypes (phenotype $L$ and phenotype $H$ ), with phenotype $H$ being a stage of progressive deterioration of phenotype $L$, which depends on the earliness with which ventilatory management begins and the degree of inflammatory compromise. However, since VMI can generate VILI, the use of protective ventilation has been recommended as a ventilatory strategy for $\mathrm{CO}$ VID-19. This review aims to comment on the available evidence of the essential aspects of protective IMV in the context of ARDS associated with COVID-19, in addition to the use of neuromuscular blockade and prone strategies.
\end{abstract}

\section{RESUMEN}

EI SARS-CoV-2 es el agente responsable del COVID-19, actual pandemia, que se caracteriza por desarrollar alteraciones respiratorias que cursan con hipoxemia severa asociada a cuadros de neumonía no bacteriana, SDRA hasta la falla

\section{Key words:}

SARS-CoV-2,

COVID-19,

acute lung injury,

mechanical ventilation

\section{Palabras clave:}

SARS-CoV-2,

COVID-19,

Insuficiencia respiratoria aguda,

ventilación mecánica

Médico Cirujano, Medicina de Urgencia Adulto, Hospital Clínico Universidad de Chile.

Enfermera Universitaria, Hospital Clínico Universidad de Chile.

Interno de Medicina, Universidad San Sebastián.

Fecha de recepción: 09 de mayo de 2020

Fecha de aceptación: 30 de mayo de 2020

\section{ORCID}

https://orcid.org/0000-0003-4156-610X

Financiamiento y Conflicto de Interés

El informe de los autores no muestra ningún conflicto de interés ni hubo fuentes externas de financiación.

Correspondencia:

Javier García Garzón

Email: fds.baar@gmail.com 
multiorgánica. Se ha caracterizado por presentar 2 fenotipos distintos (fenotipo $\mathrm{L}$ y fenotipo $\mathrm{H}$ ), siendo el fenotipo $\mathrm{H}$ un estadío de deterioro progresivo del fenotipo $L$, que depende de la precocidad con la que se inicia el manejo ventilatorio y del grado de compromiso inflamatorio. Sin embargo, dado que la VMI puede generar VILI, se ha recomendado el uso de una ventilación protectora como estrategia ventilatoria para COVID-19. La presente revisión tiene como objetivo comentar la evidencia disponible de los aspectos esenciales de la VMI protectora en el contexto del SDRA asociado a COVID-19, además del uso de bloqueo neuromuscular y las estrategias de prono.

\section{Introducción}

- I coronavirus 2 del síndrome respiratorio agudo severo (SARS-CoV-2) es el agente causante de la enfermedad del coronavirus 2019 (COVID-19), originada en la ciudad de Wuhan (China) a fines del año 2019, actual pandemia[1]. Se caracteriza por desarrollar alteraciones respiratorias como cuadros de neumonía no bacteriana, síndrome de distrés respiratorio agudo (SDRA) y falla multiorgánica, con necesidad de terapia de soporte inminente.

La afinidad del SARS-CoV-2 depende de la interacción de una de sus proteínas estructurales (proteína S) con el receptor ECA-2 (enzima convertidora de angiotensina 2) distribuido en las vías respiratorias y las células epiteliales alveolares, formando un complejo proteína-receptor que se internaliza estas células, permitiendo la reproducción viral y su posterior exocitosis e invasión del resto de los epitelios pulmonares (repitiendo el mismo ciclo de replicación viral). En respuesta a este proceso infectivo, los macrófagos locales estimulan la liberación de citoquinas proinflamatorias que desarrollarán un síndrome de liberación de citoquinas[2]; mecanismo que mediará el grado de compromiso multiorgánico y, finalmente, la muerte de los pacientes. A nivel pulmonar, estas citoquinas promoverán un aumento de la permeabilidad vascular, facilitando la salida de plasma y células sanguíneas a los alveolos, con una vasorreactividad alterada cuya vasoplejia empeorará el fenómeno de shunt; expresándose clínicamente como una neumonía viral con daño pulmonar agudo hasta un SDRA[2],[3],[4], que cursa principalmente con hipoxemia. En compensación a la disminución del oxígeno sanguíneo, se produce un aumento de la ventilación y con ello del volumen corriente o tidal $\left(V_{t}\right)$, y frecuencia respiratoria[4]; todo esto, en contexto de un estado proinflamatorio que exacerba las necesidades metabólicas del organismo, manifestando mayor demanda y consumo de oxígeno, incrementando así el trabajo respiratorio[4].
Desde un punto de vista fenotípico, la clínica del SDRA es lo suficientemente heterogénea para presentar dificultad en su manejo, no obstante, puede definirse un patrón clínico clásico. El COVID-19, en cambio se caracteriza por cursar con una hipoxemia severa que puede ser asintomática (raramente observada en el SDRA grave), o bien, asociarse a disnea intensa, hipocapnia profunda, normocapnia o hipercapnia; o caracterizarse por responder a óxido nítrico inhalado o a la posición prono[3]. Una publicación italiana, describió una serie de posibles factores influyentes en el fenotipo del COVID-19; entre estos destacan la gravedad de la infección, la respuesta del huésped, comorbilidades, capacidad de respuesta ventilatoria a la hipoxemia, tiempo transcurrido entre el inicio de la enfermedad y la vigilancia hospitalaria de posibles complicaciones[3]. Con estos, se pudo discernir entre dos fenotipos frecuentes: fenotipo $L$ y el fenotipo $H$. El fenotipo $L$ posee una frecuencia de $70-80 \%$ y se caracteriza por cursar con edema intersticial subpleural local, con escasa imagen de opacidad en vidrio esmerilado y amplias zonas aireadas ventilando, pero con una baja relación ventilación-perfusión, una baja elastancia pulmonar (con una compliance normal) y una baja capacidad de reclutamiento. Por otro lado, el fenotipo $\mathrm{H}$ posee una frecuencia de $20-30 \%$, con un patrón semejante al SDRA clásico, caracterizado por cursar con una mayor cantidad de edema de infiltración bilateral, con menos zonas aireadas ventilando, además, de una mayor elastancia pulmonar (con una compliance disminuida), un mayor shunt de derecha a izquierda y una mayor capacidad de reclutamiento; es decir, un patrón típico de pulmón pequeño o "baby lung"[5]. Se cree que el paso de un fenotipo $L$ a un fenotipo $\mathrm{H}$ depende de la precocidad con que se inicia el manejo ventilatorio y del grado de compromiso inflamatorio que se presente, haciendo necesario conocer los criterios clínicos y medidas terapéuticas para manejar la hipoxemia[4].

La presente revisión tiene como objetivo comen- 
tar la evidencia disponible de los aspectos esenciales de la ventilación mecánica invasiva (VMI) protectora y sus recomendaciones en el manejo del SDRA dada su frecuente asociación a COVID-19, junto a la evidencia y recomendación de las estrategias de bloqueo neuromuscular y maniobras de prono. Se debe considerar que estas recomendaciones son en base a la evidencia disponible, lo que puede presentar modificaciones en el tiempo debido al estudio continuo del manejo del COVID-19.

\section{Aspectos fisiopatológicos y técnicos de la VMI}

\section{Generalidades de la VMI}

La VMI se considera una medida de soporte que busca reemplazar la función respiratoria del paciente. Para ello, debe manejar una serie de elementos físicos que incluyen fuerza (presión), desplazamiento (volumen) y velocidad de cambio en relación al tiempo (flujo)[6]. Su uso está dirigido a situaciones de insuficiencia respiratoria hipoxémica e hipercápnica, con el fin de estabilizar estos cuadros disminuyendo el trabajo inspiratorio, redistribuyendo el flujo sanguíneo de la actividad muscular respiratoria en situaciones de shock y aportando una ventilación protectora en pacientes con SDRA[6].

A grandes rasgos, se puede clasificar la ventilación según la variable establecida: volumen control (VC) y presión control (PC). En VC existen dos estrategias para secuenciar la respiración: asistido controlado (AC) y la ventilación mandatoria intermitente sincronizada (SIMV). En PC, sus estrategias son AC, SIMV, presión soporte (PS), VC regulado por presión (PRVC) y BiPAP/Bilevel (ventilación binivelada). Otra modalidad es la ventilación espontánea (ESP)[6]. En el contexto COVID-19, la modalidad recomendada es la VC-AC. El resto de las variables controlables son las presiones de vía aérea (presión plateau y presión de distención alveolar o driving pressure), el trigger, el $V_{t}$, la frecuencia respiratoria, la relación inspiración/ espiración (i/e), el flujo inspiratorio, la fracción inspirada de oxígeno $\left(\mathrm{FiO}_{2}\right)$ y el PEEP (presión positiva al final de la expiración)[6]. A partir de la interacción de estas variables, nacen las 3 curvas que se observan en los monitores de todo ventilador: volumen versus tiempo, flujo versus tiempo y presión versus tiempo. La modificación de algunos de estos parámetros ventilatorios puede generar modificaciones en la hemodinamia de los pacientes con SDRA. En este contexto, es necesario el uso de drogas vasoactivas por sobre la utilización de volumen, dado que el empleo de una mayor expansión del fluido suele ser inútil e incluso perjudicial en este tipo de pacientes[7].

Por otro lado, es importante tener presente que la VMI no es inocua, pudiendo generar daño asociado a la ventilación mecánica (VILI)[4],[8] mediante mecanismos de daño traumático que pueden resumirse en tres tipos: Volutrauma (por exceso de $V_{t}$ aportado, generando strain o elongación de las unidades alveolares), barotrauma (por exceso de presiones elevadas en la vía aérea que generan estrés alveolar) y atelectrauma (generado por la apertura y cierre cíclico de las unidades alveolares)[8]. Conocer estos mecanismos ha permitido la creación de estrategias ventilatorias controladas que eviten el VILI; introduciendo el concepto de ventilación protectora.

\section{Estrategia ventilatoria protectora}

Es una estrategia ventilatoria que ha mostrado resultados positivos en distintos escenarios clínicos, reduciendo las complicaciones pulmonares y la mortalidad. Se presume un método efectivo para el manejo del COVID-19, por lo que es la terapia actualmente empleada[4],[9],[10]. Se recomienda la modalidad VC-AC, ya que, es simple de ejecutar y permite su manejo por operadores no expertos. Esta se emplea en base al ajuste de variables como $V_{t}$, frecuencia respiratoria, relación i/e, $\mathrm{FiO}_{2}$, presión de vía aérea (presión plateau y driving pressure) y la titulación de PEEP (Figura 1).

En el año 2000, un estudio comparó una estrategia con $V_{t}$ bajos (6 ml/peso ideal) versus $V_{t}$ tradicionales (12 ml/peso ideal) en pacientes con SDRA, demostrando una mortalidad de $31 \%$ y $12 \%$ respectivamente[11]. Esta reducción en la mortalidad también se observó en otros escenarios, donde el empleo de $V_{t}$ bajos evidenció un menor número de complicaciones tanto pulmonares como extrapulmonares[12]. Por tanto, se entiende que el $V_{t}$ es un pilar fundamental en la ventilación protectora. Las recomendaciones actuales sugieren un $V_{t}$ de 6 - $8 \mathrm{ml} /$ peso ideal, considerando el peso ideal (calculado en función de la talla del paciente, la que difiere entre hombres y mujeres) (Figura 1). Esta consideración de peso ideal se debe a que el volumen pulmonar no se modifica con el peso real, pero sí con la superficie corporal. Así, se pretende entregar el $V_{t}$ necesario que requiere un pulmón aireado, pues un $V_{t}$ demasiado bajo puede conducir a hipoventilación y atelectasia[4].

La frecuencia respiratoria debe ajustarse con el objetivo de evitar una acidosis respiratoria, por lo que su monitoreo dependerá del control gasométrico. En este sentido, se ajustará la frecuencia a partir de un 


\begin{tabular}{|c|c|c|c|c|c|}
\hline \multirow[b]{2}{*}{ Modalidad: Volumen Control (A/C) } & \multicolumn{2}{|c|}{$\begin{array}{l}\text { Titulación: Baja } \\
\text { PEEP/Alta } \mathrm{FiO}_{2}\end{array}$} & & \multicolumn{2}{|c|}{$\begin{array}{l}\text { Titulación: Alto } \\
\text { PEEP/Baja } \mathrm{FiO}_{2}\end{array}$} \\
\hline & PEEP & $\mathrm{FiO}_{2}$ & & PEEP & $\mathrm{FiO}_{2}$ \\
\hline \multirow{2}{*}{ Volumen Corriente: $V_{\mathrm{t}}=6-8 \mathrm{ml} /$ peso ideal } & 5 & 0,3 & & 5 & 0,3 \\
\hline & 5 & 0.4 & & 8 & 0,3 \\
\hline FR: $20-25 \mathrm{rpm}\left(\mathrm{PaCO}_{2}<60 \mathrm{mmHg}\right.$ y pH $\left.>7,22\right)$ & 8 & 0,4 & & 10 & 0,3 \\
\hline \multirow[t]{2}{*}{ Relación i/e:1:2 (aumentar en obstrucción) } & 8 & 0.5 & & 10 & 0.4 \\
\hline & 10 & 0,5 & & 12 & 0,4 \\
\hline $\mathrm{FiO}_{2}$ : Inicial $100 \%$ y titular $p$ ara $\mathrm{SpO}_{2}=88-93 \%$ & 10 & 0.6 & & 14 & 0.4 \\
\hline \multirow[t]{2}{*}{ Pausa Inspiratoria: $0,2-0,5$ segundos } & 10 & 0,7 & & 16 & 0,4 \\
\hline & 12 & 0,7 & \multirow{7}{*}{ 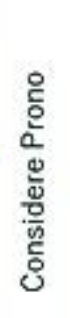 } & 18 & 0,4 \\
\hline \multirow{2}{*}{$\begin{array}{r}\text { Metas de Presión: Presión Plateau } \leq 28 \mathrm{cmH}_{2} \mathrm{O} \\
\text { Driving Pressure } \leq 14 \mathrm{cmH}_{2} \mathrm{O}\end{array}$} & 14 & 0,7 & & 18 & 0,5 \\
\hline & 14 & 0,8 & & 20 & 0,5 \\
\hline \multirow[t]{2}{*}{ PEEP: Iniciar con $8-12 \mathrm{cmH}_{2} \mathrm{O}$ (Titular según tabla) } & 14 & 0,9 & & 20 & 0,6 \\
\hline & 16 & 0,9 & & 20 & 0,7 \\
\hline \multirow{2}{*}{$\begin{array}{l}\text { Peso ideal of: } 50+0,91 \cdot(\text { talla }-152,4) \\
\text { Peso ideal } \$: 45,5+0,91 \cdot(\text { talla }-152,4)\end{array}$} & 18 & 0.9 & & 20 & 0,8 \\
\hline & $18-24$ & 1,0 & & $22-24$ & $0,8-1,0$ \\
\hline
\end{tabular}

Figura 1. Estrategia de ventilación protectora. El ajuste de VMI protectora debe hacerse según los parámetros indicados en esta Tabla; utilizando un sistema de aspiración cerrado, con Vt bajos, evitando presiones plateau por encima de $30 \mathrm{~cm}$ de $\mathrm{H}_{2} \mathrm{O}$ y un driving pressure por debajo de $15 \mathrm{~cm}$ de $\mathrm{H}_{2} \mathrm{O}$ (menor mortalidad). La titulación del PEEP debe iniciarse en rangos fisiológicos cercanos a $5 \mathrm{cmH}_{2} \mathrm{O}$, ajustándolo según tabla de titulación de baja PEEP y alta $\mathrm{FiO}_{2}$ (en función de la compliance, oxigenación, espacio muerto y estado hemodinámico). Debe mantenerse un balance hídrico negativo para evitar un exceso de edema en el pulmón. Considere el uso de maniobras de pronación de al menos 16 horas, si PaFi < 150 (pese a administrar altas concentraciones de $\mathrm{O}_{2}$ ) o ante necesidad de uso de rangos de PEEP altos, asociado a bloqueo neuromuscular para evitar casos de asincronía con el ventilador.

valor inicial de 20 respiraciones por minuto (rpm), manteniendo una hipercapnia leve permisiva con una $\mathrm{PaCO}_{2}<60 \mathrm{mmHg}$ que no produzca una acidosis significativa (pH > 7,3)[11],[13]. Dado que el fenotipo L del COVID-19 no cursa compromiso hemodinámico inicial, es posible permitir estos parámetros de $\mathrm{PaCO}_{2}$ y $\mathrm{pH}$ sin producir descompensación hemodinámica. De este modo, se intenta otorgar la menor cantidad de ventilaciones posibles evitando generar daño por exceso de frecuencia respiratoria. Su ajuste, debe hacerse con una relación i/e de 1:2 (la que puede aumentarse a 1:3, 1:4 y 1:5 en aquellos pacientes que muestren clínica obstructiva), y con una fracción inspirada de oxígeno $\left(\mathrm{FiO}_{2}\right)$ que, si bien puede iniciarse al 100\% durante la intubación, una vez estable el intercambio gaseoso, se debe titular de forma precoz a metas conservadoras para una saturación entre 88 a 94\%, evitando tanto la hipoxemia como la hiperoxia (Figura 1)[14].

Las distintas presiones de la vía aérea en una VCAC (Figura 2) son representadas por: presión peak (representa la resistencia del pulmón y de la vía aérea superior, incluyendo la resistencia del tubo endotraqueal o TOT y la del ventilador), presión plateau (se obtiene al realizar una pausa inspiratoria de 0,25 segundos, con ello elimina la resistencia del TOT, reflejando la presión máxima que el ventilador le puede otorgar al parénquima pulmonar, permitiendo una distribución uniforme del flujo aéreo en el pulmón, estimando de forma indirecta la presión alveolar) y driving pressure (obtenida de la diferencia entre la presión plateau y el PEEP)[6]. Tanto la presión plateau como el driving pressure permiten evaluar las presiones de vía aérea. Un estudio retrospectivo de pacientes con SDRA, mostró una correlación entre el driving pressure y la mortalidad, comparándola entre tres grupos: un primer grupo de pacientes con igual PEEP mostró mayor mortalidad a mayor presión plateau (mayor driving pressure); el segundo grupo con la misma presión plateau mostró menor mortalidad en aquellos con mayor PEEP (menor driving pressure). Finalmente, un tercer grupo con distintos niveles de presión de vía aérea, pese al aumento del PEEP y la presión plateau (manteniendo un driving pressure 


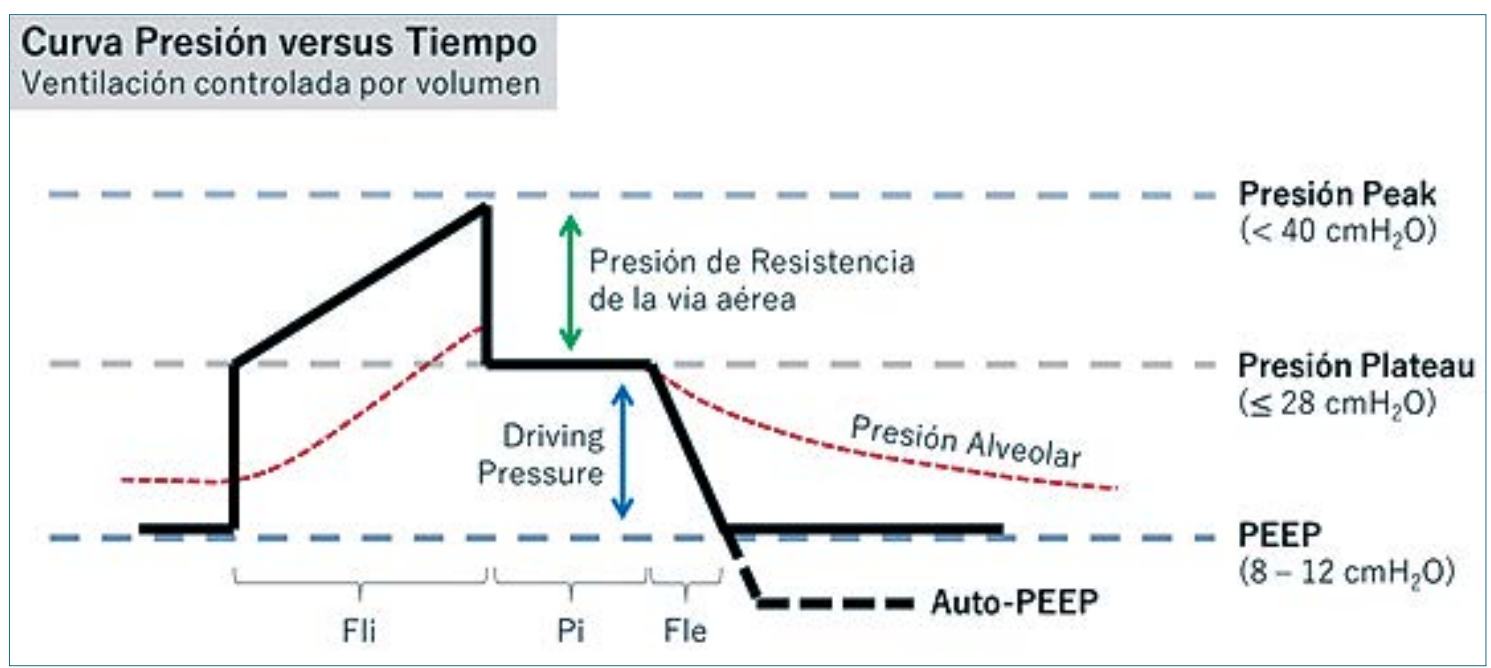

Figura 2. Interpretación de las curvas ventilatorias en volumen control. Abreviaturas: Fle: flujo espiratorio; Fli: flujo inspiratorio; Pi: presión inspiratoria.

constante), la mortalidad se mantuvo sin cambios[15]. Este estudio permitió determinar un nivel umbral de driving pressure a partir del cual la mortalidad aumenta de forma significativa, equivalente a $15 \mathrm{cnH}_{2} \mathrm{O}$. En este sentido, las recomendaciones sugeridas para el manejo de las presiones de vía aérea son una presión plateau $28 \mathrm{cmH}_{2} \mathrm{O}$ (idealmente un valor $<25 \mathrm{cmH}_{2} \mathrm{O}$ ) y un driving pressure $14 \mathrm{cmH}_{2} \mathrm{O}[15]$. Incluso, si se logra un manejo con driving pressure $<12 \mathrm{cmH}_{2} \mathrm{O}[15]$, podría manejarse un $\mathrm{V}_{t}$ de hasta $8 \mathrm{ml} /$ peso ideal, con el fin de evitar la hipoventilación.

Respecto al PEEP, se ha visto beneficio el aplicar maniobras de reclutamiento con presiones de PEEP bajas en la mayoría de las unidades reclutables. Su uso busca disminuir el daño por apertura y cierre cíclico alveolar, mejorando paralelamente la oxigenación pulmonar[14],[16]. Utilizar niveles de PEEP elevados puede generar VILI por sobredistensión (aumento del estrés y strain alveolar), alterando la oxigenación y asociando compromiso hemodinámico. Cada paciente requerirá de niveles de PEEP distintos, por lo que su uso requerirá de titulación con estrategias de bajo PEEP (Figura 1)[14],[16].

\section{Monitorización de la VMI}

Los elementos que permiten realizar una monitorización de la terapia ventilatoria son la gasometría arterial, la relación $\mathrm{PaFi}\left(\mathrm{PaO}_{2} / \mathrm{FiO}_{2}\right)$ y los parámetros límites de la ventilación protectora[17],[18],[19]. Con la medición de la gasometría arterial, se pueden plantear objetivos de $\mathrm{PaO}_{2}$ de $60-80 \mathrm{mmHg}$ (evitando tanto la hipoxemia como la hiperoxia), una $\mathrm{PaCO}_{2}<60 \mathrm{mmHg}$ (hipercapnia permisiva) y un $\mathrm{pH}$ $>7,3[4],[10],[11],[13],[14]$. El uso del índice PaFi permite evaluar la progresión de la insuficiencia respiratoria en base a los criterios clásicos de Berlín, clasificándola en hipoxemia leve (PaFi 300 - 200, con PEEP $5 \mathrm{cmH}_{2} \mathrm{O}$ ), moderada (PaFi 200 - 100, con PEEP 5 $\mathrm{cmH}_{2} \mathrm{O}$ ) y severa (PaFi 100, con PEEP $10 \mathrm{cmH}_{2} \mathrm{O}$ )[18]. Finalmente, respecto a los límites de ventilación protectora, la curva de presión versus tiempo del ventilador (Figura 2) puede otorgar información relevante respecto a los cambios en la condición clínica del paciente. Por ejemplo, una elevación de la presión peak, con una presión plateau normal, puede traducirse en un aumento de la resistencia de la vía aérea secundario a un TOT acodado o en posición monobronquial, paciente mordiendo el tubo, presencia de tapón mucoso o broncoespasmos; los cuales se manejan acomodando el TOT, manejando las secreciones de vía aérea u optimizando la sedación. Por otro lado, una elevación de la presión plateau, con una presión peak normal, puede traducirse en una disminución de la compliance pulmonar por compromiso de la pared torácica o pulmonar, como se observa en un SDRA, tubo monobronquial o un neumotórax (cuyo manejo requiere de la disminución del $V_{t}$ a 5 - $4 \mathrm{ml} /$ peso ideal o de un aumento de la frecuencia respiratoria)[19]. Por último, si el PEEP medido es mayor al PEEP seteado; con un flujo espiratorio que no alcanza a llegar a $0 \mathrm{cmH}_{2} \mathrm{O}$, esta situación se entendería como un auto- 
PEEP, la que ocurre una vez que el alvéolo no se vacía adecuadamente (consecuencia del uso de frecuencias respiratorias altas, $V_{t}$ altos, velocidades de flujo bajas e incluso del uso de presiones inspiratorias elevadas), conduciendo a un aumento de los volúmenes pulmonares al final de la espiración[6]; cuya causa puede traducirse en una obstrucción de la vía aérea (Figura 2).

En caso, una caída de la saturación de oxígeno, no se aconseja desconectar al paciente del ventilador por el riesgo de aerosolización. En este caso, es prudente evaluar la posición del TOT, descartar desconexión de la red de oxígeno o del circuito eléctrico, descartar compromiso obstructivo de la vía aérea, posibles complicaciones (neumotórax, derrame pleural, patología abdominal restrictiva, etc.) o la sobreventilación por tiempo espiratorios cortos (fenómeno que se puede revertir con broncodilatación, una disminución de la frecuencia respiratoria y aumento del tiempo espiratorio)[19].

Existen criterios de severidad (Tabla 1) que permitirán evaluar la necesidad de nuevos manejos en coadyuvancia a la terapia ventilatoria. Estos son el bloqueo neuromuscular y la técnica de pronación.

\section{Bloqueo neuromuscular}

Su objetivo es evitar la disincronía ventilatoria y/o la ventilación espontánea en etapas precoces, dirigido a pacientes con SDRA moderado a severo con PaFi $<150$ las primeras 48 horas; pudiendo prolongarse frente a disincronías marcadas. Un estudio mostró que el uso de bloqueo neuromuscular reducía el grado de inflamación en el SDRA versus aquello que no fue empleado[20]. Otro estudio utilizó un modelo randomizado con 339 pacientes empleando cisatracurio; observándose una mortalidad de $32 \%$ con el uso de este fármaco versus $42 \%$ con el uso de placebo[21]. Un tercer estudio comparó el uso de cisatracurio versus uso de sedación habitual en pacientes con SDRA de moderado a grave manejados con estrategia de PEEP alta. Si bien, no mostró diferencias significativas en la mortalidad entre ambos grupos[22], el uso de bloqueo neuromuscular es una recomendación vigente en pacientes en VMI[4],[10]. Los bloqueadores neuromusculares disponibles en Chile son el atracurio, cisatracurio y el rocuronio. Es importante evaluar diariamente la necesidad de mantención bloqueo neuromuscular en infusión, dado que su beneficio puede contradecirse por complicaciones como la miopatía y la polineuropatía del paciente crítico cuando son usados por períodos prolongados o en contexto de un shock séptico[23].

\section{Técnica de prono}

En un SDRA, al adoptar la posición supina, con frecuencia se desarrollan atelectasias en las regiones pulmonares dorsales debido a la gravedad, la compresión del corazón y el diafragma. La distribución de la ventilación en esta posición se concentra en las zonas no dependientes (zonas ventrales), mientras que en posición prono existe una distribución homogénea de la ventilación, mejorando la aireación en las zonas dorsales (regiones mejor perfundidas del pulmón), y con ello la relación ventilación-perfusión[24]. Un estudio experimental, comparó la ventilación-perfusión tanto en posición supina como en prono. En él, se pudo observar que la distribución de la perfusión en posición supina frecuentaba las zonas dorsales y la ventilación se distribuía hacia las zonas no dependientes del pulmón; mientras que, en posición prono, la distribución de la ventilación-perfusión se hacía más homogénea[25]. La posición prono mejora el reclutamiento pulmonar, disminuyendo la inestabilidad alveolar y la hiperinflación observada en las estrategias de PEEP alta en pacientes con SDRA, evitando el VILI al homogenizar, además, la energía aportada por el ventilador[26],[27]. Como experiencia clínica, otro estudio con 466 pacientes con SDRA, comparó el uso de ventilación protectora en posición prono y en posición supina, mostrando una mortalidad de $23,6 \%$ y $41 \%$ respectivamente. Lo destacable de este estudio, fue el tiempo de posición prono de a lo menos 16 horas, que si bien, no existe una correlación concreta con el tiempo, se cree que probablemente sea la respuesta a esta diferencia de mortalidad[28]. La experiencia con SDRA graves por otras causas sugiere

\begin{tabular}{ll}
\multicolumn{2}{c}{ Tabla 1. Criterios de severidad del monitoreo en } \\
VMI
\end{tabular}

Abreviaturas: PaFi: relación $\mathrm{PaO}_{2} / \mathrm{FiO}_{2}$; PEEP: presión positiva al final de la expiración. Adaptado de Möhlenkamp et al., Gattinoni et al., Poston et al., Brower et al., Baig et al., Meade et al., Amato et al., Ranieri et al., Richter et al., Ghelichkhani et al. 
que el uso de prono debe aplicarse precozmente. Si bien, no existe evidencia sustentable en COVID-19, se cree que éste afecta las áreas periféricas y dorsales de los pulmones, lo que propicia condiciones ideales para sugerir esta técnica[5],[25]. En este contexto, se recomiendan las maniobras de prono de forma precoz y mantenidas por 48 a 72 horas en todo paciente con SDRA por COVID-19 que curse con PaFi < 150 o uso de PEEP $>12 \mathrm{cmH}_{2} \mathrm{O}$, en contexto de una ventilación protectora asociada al bloqueo neuromuscular. Estas 3 estrategias conducen a una mejora en la oxigenación y la supervivencia de los pacientes con SDRA[25],[26],[27],[28],[29].

\section{Recomendaciones generales para el manejo ventilatorio en COVID-19}

Se considerará la intubación endotraqueal en todo paciente que curse con aumento del trabajo respiratorio, asociado a taquipnea persistente (> $30 \mathrm{rpm})$, hipoxemia refractaria $\left(\mathrm{SpO}_{2}<90 \%\right)$ pese a oxígeno suplementario $\left(\mathrm{FiO}_{2} 50 \%\right)$, insuficiencia respiratoria aguda y criterios de shock[30]. Dado que la infección por SARS-CoV-2 tiene una tasa de transmisibilidad muy elevada[31], la intubación de los pacientes con COVID-19 se considera una técnica de alto riesgo de transmisión viral hacia el equipo de salud[5]. Esto justifica el uso de salas de aislamiento de infecciones transmitidas por el aire[32], junto la asignación del operador más capacitado para realizar el procedimiento de intubación. Este debe disponer de los elementos de protección personal (EPP) junto a un número limitado de asistentes (idealmente dos médicos, un enfermero/a y un técnico en enfermería), de tal modo reducir el grado de exposición del personal al realizar el manejo de vía aérea[5],[32],[33]. Puesto que el mecanismo de transmisión del SARS-CoV-2 es por gotitas y contacto; los EPP incluyen guantes, delantal desechable con manga larga, protección ocular (antiparras o visores integrales) y mascarilla[32]. Se aconseja utilizar mascarilla N95, desplazando a las mascarillas quirúrgicas en la atención y asistencia del resto de procedimientos. La higiene de las manos es esencial antes de ponerse y después de quitarse el EPP[32].

Considere la intubación precoz programada, evitando una intubación de emergencia, dado que esta última situación no asegura el tiempo requerido para la correcta aplicación de los EPP. Para minimizar el uso de ventilaciones manuales, se recomienda la oxigenación previa con $\mathrm{FiO}_{2}$ al $100 \%$ durante 5 minutos. El uso de máscara de bolsa (ambú) es una maniobra generadora de aerosoles, por lo cual, es importante garantizar la instalación de un filtro HMEF (intercambiador de humedad y temperatura con capacidad de filtro) o HEPA (filtro de alta eficacia de partículas aéreas) para evitar la dispersión del SARS-CoV-2, manipulando estas ventilaciones en una sala con presión negativa (en caso de estar disponible) o una habitación cerrada[33],[34]. Tras la hiperoxigenación, se realizará una secuencia rápida de intubación, en lo posible con videolaringoscopía (dado que permite mantener distancia física), previa administración de sedantes, hipnóticos y bloqueador muscular. La confirmación de la correcta colocación del TOT se realiza mediante la auscultación de campos pulmonares, capnografía y radiografía de tórax[5]. Una vez intubado el paciente, es importante asegurar el correcto monitoreo de la VMI programando parámetros de modalidad de ventilación protectora (según peso ideal y sexo del paciente) junto con la activación de las alarmas respectivas[5]. En caso de presentar parámetros de alto riesgo a la evaluación de la severidad (Tabla 1), considere la aplicación de bloqueo neuromuscular o posición prono, asegurando la asesoría de personal especialista.

Es importante verificar ciertos aspectos técnicos como el acceso a una red de oxígeno y red de aire, conexión eléctrica, correcta desinfección del ventilador con amonio cuaternario o alcohol isopropílico al $70 \%$, instalación estéril del circuito ventilatorio y la realización de un test de ATC (compensación automática del TOT) con el fin de asegurar presiones adecuadas del sistema[34]. Los ventiladores mecánicos poseen 2 accesos (inspiratorio y espiratorio) que se fusionan a través de un enramado y forman un sistema cerrado que permite medir la cantidad de aire que entra y sale en relación al tiempo. Entre este enramado y el TOT debe verificarse la conexión de los filtros virales; además, de la utilización de sistemas de aspiración cerrada, el retiro de vaporizadores para el uso de ventilación prolongada y disminuir las desconexiones a lo mínimo posible[33]. Estas son conductas que disminuyen y previenen el riesgo de formación de aerosoles.

A la fecha, existen ensayos en la búsqueda de opciones más estrictas de VMI para el manejo de COVID-19, bajo el concepto de ventilación ultraprotectora con un $V_{t}$ ultra bajo $(\leq 4,2 \mathrm{ml} /$ peso ideal) $\sin$ circulación extracorpórea, con una reducción media de $4 \mathrm{cmH}_{2} \mathrm{O}$ en el driving pressure, bajo la hipótesis de que podría reducir la mortalidad a partir del día 90 y aumentar el número de días sin VMI a partir del día 60 , en comparación con la ventilación protectora actualmente difundida[35]. 


\section{Conclusiones}

El SARS-CoV-2 es el agente causante del COVID19, caracterizado por desarrollar alteraciones respiratorias que cursan con hipoxemia severa asociada a cuadros de neumonía no bacteriana, SDRA hasta la falla multiorgánica; mediado por un mecanismo inmunológico dependiente de citoquinas. El COVID-19 posee 2 fenotipos particulares, fenotipo $L$ y fenotipo $\mathrm{H}$, siendo el fenotipo $\mathrm{H}$ un estadío de deterioro progresivo del fenotipo $L$, que depende de la precocidad con que se inicia el manejo ventilatorio y del grado de compromiso inflamatorio. No obstante, dado que la VMI puede generar VILI, se ha recomendado el uso de una ventilación protectora como estrategia ventilatoria. Esta se caracteriza por el uso de Vt bajos $(6-8 \mathrm{ml} /$ peso ideal) asociado a parámetros ajustados de frecuencia respiratoria (20 rpm), con una relación i/e de $1: 2$, una $\mathrm{FiO}_{2}$ titulada con PEEP y presiones de vía aérea bajas (presión plateau $28 \mathrm{cmH}_{2} \mathrm{O}$ y driving pressure $14 \mathrm{cmH}_{2} \mathrm{O}$ ). El monitoreo de la VMI dependerá de la gasometría arterial, la relación PaFi y los parámetros límites que se programen en el ventilador; los que permitirán decidir el inicio de manejos coadyuvantes como el bloqueo neuromuscular y la técnica de prono. Ambas medidas tienen evidencia suficiente que reportan disminución de la mortalidad y mejoría de la ventilación perfusión, optimizando la oxigenación y la supervivencia de los pacientes con SDRA.

Dada la alta tasa de transmisibilidad del SARSCoV-2, el proceso de intubación precoz programada debe realizarse en una sala de aislamiento de infecciones transmitidas por el aire y por un operador capacitado, con un número limitado de asistentes. Todos deberán disponer de los EPP suficientes con el fin de reducir el grado de exposición. Importante es, además, la verificación de la instalación de filtros antivirales, asegurando el correcto monitoreo de la VMI junto con la activación de las alarmas respectivas. Si bien, estas medidas son conductas que disminuyen y previenen el riesgo de formación de aerosoles, es probable que los protocolos cambien según la evidencia que se vaya reportando.

\section{Referencias}

1. Zhu N, Zhang D, Wang W, Li X, Yang B, Song J, et al. A novel coronavirus from patients with pneumonia in China, 2019. N Engl J Med. 20 de febrero de 2020 [citado 6 de mayo de 2020];382(8):727-33. http:// www.nejm.org/doi/10.1056/NEJMoa2001017

2. Li X, Geng M, Peng $Y$, Meng L, Lu S. Molecular immune pathogenesis and diagnosis of $\mathrm{CO}$ VID-19. Journal of Pharmaceutical Analysis. Elsevier BV; 2020 Apr;10(2):102-8. http://dx.doi. org/10.1016/j.jpha.2020.03.001

3. Gattinoni L, Chiumello D, Caironi P, Busana M, Romitti F, Brazzi L, et al. COVID-19 pneumonia: different respiratory treatments for different phenotypes? Intensive Care Medicine. Springer Science and Business Media LLC; 2020 Apr 14;46(6):1099-102. http:// dx.doi.org/10.1007/s00134-020-

\section{3-2}

4. Möhlenkamp S, Thiele H. Ventilation of COVID-19 patients in intensive care units. Herz. Springer Science and Business Media LLC; 2020 Apr 20;45(4):329-

31. http://dx.doi.org/10.1007/ s00059-020-04923-1.

5. Gattinoni L, Pesenti A. The concept of "baby lung". Intensive Care Med. 6 de junio de 2005;31(6):776-84.

6. Walter JM, Corbridge TC, Singer BD. Invasive Mechanical Ventilation. South Med J. 1 de diciembre de 2018;111(12):74653. https://doi.org/10.14423/ SMJ.0000000000000905.

7. Cortés-Puentes GA, Oeckler RA, Marini JJ. Physiology-guided management of hemodynamics in acute respiratory distress syndrome. Ann Transl Med. septiembre de 2018;6(18):353353. https://doi.org/10.21037/ atm.2018.04.40.

8. Tidal volume is a major deter- minant of cyclic recruitmentderecruitment in acute respiratory distress syndrome - Minerva Anestesiologica 2011 April;77(4):418-26 - Minerva Medica - Journals. https://www. minervamedica.it/en/journals/ minerva-anestesiologica/article. php?cod=R02Y2011N04A0418

9. Phua J, Weng $L$, Ling $L$, Egi M, Lim C-M, Divatia JV, et al. Intensive care management of coronavirus disease 2019 (COVID-19): challenges and recommendations. The Lancet Respiratory Medicine. Elsevier BV; 2020 May;8(5):506-17. http://dx.doi.org/10.1016/s22132600(20)30161-2

10. Poston JT, Patel BK, Davis AM. Management of Critically III Adults With COVID-19. JAMA [Internet]. American Medical Association (AMA); 2020 Mar 26. http://dx.doi.org/10.1001/ jama.2020.4914

11. Brower RG, Matthay MA, Morris 
A, Schoenfeld D, Thompson

BT, Wheeler A. Ventilation

with Lower Tidal Volumes as

Compared with Traditional

Tidal Volumes for Acute Lung

Injury and the Acute Respiratory

Distress Syndrome. N Engl J

Med [Internet]. 4 de mayo de

2000;342(18):1301-8. http://

www.nejm.org/doi/abs/10.1056/

NEJM200005043421801

12. Futier $\mathrm{E}$, Constantin JM, Paugam-Burtz C, Pascal J, Eurin M, Neuschwander A, et al. A Trial of Intraoperative Low-Tidal-Volume Ventilation in Abdominal Surgery. N Engl J Med. 31 de agosto de 2013;369(5):428-37. Disponible en: http://www.nejm.org/ doi/10.1056/NEJMoa1301082

13. Baig MA. The COVID-19 Intubation and Ventilation Pathway (CiVP); a Commentary. Arch Acad Emerg Med. 2020;8(1):e37. http://www.ncbi. nlm.nih.gov/pubmed/32232219

14. Meade MO, Cook DJ, Guyatt $\mathrm{GH}$, Slutsky AS, Arabi YM, Cooper DJ, et al. Ventilation strategy using low tidal volumes, recruitment maneuvers, and high positive end-expiratory pressure for acute lung injury and acute respiratory distress syndrome: A randomized controlled trial. JAMA - J Am Med Assoc. 13 de febrero de 2008;299(6):63745. https://doi.org/10.1001/ jama.299.6.637.

15. Amato $M B$, Meade $M O$, Slutsky AS, Brochard L, Costa EL, Schoenfeld DA, et al. Driving Pressure and Survival in the Acute Respiratory Distress Syndrome. N Engl J Med. 19 de febrero de 2015;372(8):747-55. http:// www. nejm.org/doi/10.1056/NEJMsa1410639

16. Gattinoni L, Caironi P, Cressoni $\mathrm{M}$, Chiumello $\mathrm{D}$, Ranieri VM, Quintel M, et al. Lung Recruitment in Patients with the Acute Respiratory Dis- tress Syndrome. N Engl J

Med [Internet]. 27 de abril de

2006;354(17):1775-86. http://

www.nejm.org/doi/abs/10.1056/ NEJMoa052052

17. Marini JJ, Gattinoni L. Management of COVID-19 Respiratory Distress. JAMA. American Medical Association (AMA); 2020 Jun 9;323(22):2329. http://dx.doi. org/10.1001/jama.2020.6825

18. Ranieri VM, Rubenfeld GD, Thompson BT, Ferguson ND, Caldwell E, Fan E, et al. Acute respiratory distress syndrome: The Berlin definition. JAMA - J Am Med Assoc. 13 de junio de 2012;307(23):2526-33.

19. Pérez M, Mancebo J. Monitoring ventilatory mechanics. Med Intensiva. 1 de diciembre de 2006;30(9):440-8.

20. Forel JM, Roch A, Marin V, Michelet P, Demory D, Blache JL, et al. Neuromuscular blocking agents decrease inflammatory response in patients presenting with acute respiratory distress syndrome*. Crit Care Med [Internet]. noviembre de 2006 [citado 6 de mayo de 2020];34(11):2749-57. Disponible en: http://journals.Iww. com/00003246-20061100000007

21. Papazian L, Forel JM, Gacouin A, Penot-Ragon C, Perrin G, Loundou A, et al. Neuromuscular Blockers in Early Acute Respiratory Distress Syndrome. N Engl J Med [Internet]. 16 de septiembre de 2010 [citado 6 de mayo de 2020];363(12):1107-16. Disponible en: http://www.nejm.org/doi/ abs/10.1056/NEJMoa1005372

22. Moss $M$, Huang DT, Brower RG, Ferguson ND, Ginde AA, Gong $M N$, et al. Early Neuromuscular Blockade in the Acute Respiratory Distress Syndrome. N Engl J Med [Internet]. 23 de mayo de 2019 [citado 6 de mayo de 2020];380(21):1997-2008.
Disponible en: http://www. nejm.org/doi/10.1056/NEJMoa1901686

23. Price D, Kenyon NJ, Stollenwerk N. A fresh look at paralytics in the critically ill: Real promise and real concern. Vol. 2, Annals of Intensive Care. Springer Verlag; 2012. p. 43.

24. Henderson AC, Sá RC, Theilmann RJ, Buxton RB, Prisk GK, Hopkins SR. The gravitational distribution of ventilationperfusion ratio is more uniform in prone than supine posture in the normal human lung. J Appl Physiol [Internet]. 1 de agosto de 2013 [citado 6 de mayo de 2020];115(3):313-24. Disponible en: https://www.physiology. org/doi/10.1152/japplphysiol.01531.2012

25. Richter T, Bellani G, Harris RS, Melo MFV, Winkler T, Venegas $J G$, et al. Effect of prone position on regional shunt, aeration, and perfusion in experimental acute lung injury. Am J Respir Crit Care Med. 15 de agosto de 2005;172(4):480-7. https:// doi.org/10.1164/rccm.2005010040C.

26. Cornejo RA, Díaz JC, Tobar EA, Bruhn AR, Ramos CA, González $R A$, et al. Effects of prone positioning on lung protection in patients with acute respiratory distress syndrome. Am J Respir Crit Care Med [Internet]. 15 de agosto de 2013 [citado 6 de mayo de 2020];188(4):440-8. Disponible en: http://www. atsjournals.org/doi/abs/10.1164/ rccm.201207-12790C https:// doi.org/10.1164/rccm.201207$12790 \mathrm{C}$.

27. Mentzelopoulos SD, Roussos C, Zakynthinos SG. Prone position reduces lung stress and strain in severe acute respiratory distress syndrome. Eur Respir J. 1 de marzo de 2005;25(3):534-44. https://doi.org/10.1183/0903193 
6.05.00105804.

28. Guérin C, Reignier J, Richard JC, Beuret P, Gacouin A, Boulain T, et al. Prone Positioning in Severe Acute Respiratory Distress Syndrome. N Engl J Med [Internet]. 6 de junio de 2013 [citado 6 de mayo de 2020];368(23):215968. Disponible en: http://www. nejm.org/doi/10.1056/NEJMoa1214103

29. Ghelichkhani P, Esmaeili M. Prone Position in Management of COVID-19 Patients; a Commentary. Arch Acad Emerg Med [Internet]. 2020 [citado 6 de mayo de 2020];8(1):e48. Disponible en: http://www.ncbi.nlm. nih.gov/pubmed/32309812

30. Yao W, Wang $T$, Jiang $B, G a o$ F, Wang L, Zheng H, et al.; collaborators. Emergency tracheal intubation in 202 patients with COVID-19 in Wuhan,
China: lessons learnt and international expert recommendations. Br J Anaesth. 2020 Jul;125(1):e28-37. https://doi. org/10.1016/j.bja.2020.03.026 PMID:32312571

31. Sun J, He W-T, Wang L, Lai A, Ji X, Zhai X, et al. COVID-19: Epidemiology, Evolution, and CrossDisciplinary Perspectives. Trends in Molecular Medicine. Elsevier BV; 2020 May;26(5):483-95. http://dx.doi.org/10.1016/j. molmed.2020.02.008

32. Coronavirus (2019-nCoV) COVID-19 [Internet]. [citado 6 de mayo de 2020]. Disponible en: https://www.asahq.org/ about-asa/governance-andcommittees/asa-committees/ committee-on-occupationalhealth/coronavirus

33. Clinical management of severe acute respiratory infection when
COVID-19 is suspected [Internet] [citado 6 de mayo de 2020]. Disponible en: https://www.who. int/publications-detail/clinicalmanagement-of-severe-acuterespiratory-infection-when-novel-coronavirus-(ncov)-infectionis-suspected

34. Jeria RA, Valverde DW, Gajardo FB, Shaaf IS, González PF. Guía de Recomendaciones de Armado y Uso de Filtros en Ventilación Mecánica COVID19. Sociedad Chilena de Medicina Intensiva. SOCHIMl; 2020. p. 33.

35. Ultraprotective Ventilation Without Extracorporeal Circulation During CO. 19 Pneumonia Full Text View - ClinicalTrials.gov https://clinicaltrials.gov/ct2/show/ NCT04349618?cond=COVID19+AND+\%22Respiratory+Distr ess+Syndrome\%2C+Adult $\% 22 \&$ draw $=5$ 